\title{
Felix Diergarten / Markus Neuwirth, Formenleh- re. Ein Lese- und Arbeitsbuch zur Instrumental- musik des 18. und 19. Jahrhunderts, Laaber: Laaber 2019
}

Schlagworte/Keywords: Analyse; analysis; compositional technique; Form; Formenlehre; historical theory of form; historische Formenlehre; instrumental music; Instrumentalmusik; Satzlehre; sonata; Sonate; theory of form

Seit dem Erscheinen der Formenlehre der Musik (1987) von Clemens Kühn, die inzwischen in elf Auflagen vorliegt und bis heute in der deutschsprachigen Hochschullehre immer noch sehr prominent ist, sind nunmehr über drei Jahrzehnte vergangen. Kühns Ansatz, den subjektorientierten Verstehensbegriff der Pädagogik der 1970er Jahre und den Dahlhaus'schen Formbegriff' $^{1}$ zu einer verallgemeinernden musikalischen Gattungspoetik zu vereinen, war damals radikal neu, bot er damit doch einem rigiden Schematismus die Stirn. Indem er dazu anregte, musikalische Formen anhand offener Kategorien sensibel und in Bezug auf die konkrete Komposition möglichst individuell zu betrachten, erreichte er ein großes Lesepublikum. Kühns Formenlehre ist heute zu einem musiktheoretischen Meilenstein geworden, der maßgeblich zu einer künstlerisch inspirierte(re)n und vielleicht auch phantasievolleren Hochschullehre und Musikvermittlung beigetragen hat.

Nun ist eine neue Formenlehre erschienen, die das Potenzial hat, Kühns Buch auf der Hitliste der musiktheoretischen Grundausbildung abzulösen, denn sie erfüllt verschiedene Desiderate, der eine sklassische Formenlehre te, zu Beginn der 2020er Jahre, nachkommen sollte. Sie hält dem Wandel und den inzwischen zentral gewordenen wissenschaftlichen Ansprüchen des Faches an deutschsprachigen Hochschulen insofern Stand, als sie dem selbstverständlichen Methodenpluralismus der letzten Jahrzehnte Rechnung trägt und dennoch eigene Wege zu einer klaren Vermittlung findet. Die Veröffentlichung eines solchen Buches erfordert Reflexion und vor allem auch Mut.
Die Autoren wählen dafür keinen systematischen, übergreifenden Formbegriff, sondern, ganz im Sinne der historischen Quellen des 18. Jahrhunderts, einen »modularen« (18-20), der an einem nachvollziehbaren handwerklichen Schaffensprozess ausgerichtet ist. Mit ausgewählten Bausteinen und typischen Eigenschaften der Kompositionen gelangt man so vom Kleinen ins Große. Dennoch sind unterschiedliche Theorien und Kategorienbildungen dort aufgezeigt, wo es sinnvoll erscheint. Meist werden sie zunächst prägnant zusammengefasst und anschaulich miteinander verglichen. Argumentation, Synthesen und Terminologie entstehen dann überwiegend aus einer gattungs- wie theoriehistorischen Perspektive heraus. Zentraler Ansatz des Buches ist, im Sinne des Handwerklichen, eine möglichst objektive, an den musikalischen Phänomenen ausgerichtete Werkbetrachtung in allgemeinverständlicher musiktheoretischer Sprache. Einem selbstreferenziellen Kreisen um schematische Formenlehrekategorien können die Autoren damit erfolgreich entgegenwirken. Drei Richtungen sind in den Darstellungen explizit vertreten:

1. die bekannten deutschsprachigen Traditionen, verwurzelt im 19. und frühen 20. Jahrhundert, zurückgehend auf Adolph Bernhard Marx und Erwin Ratz (respektive Arnold Schönberg);

2. jüngere angloamerikanische Ansätze einer `New Formenlehre`, vornehmlich vertreten durch William E. Caplin (ausgerichtet an Ratz/Schönberg und Carl Dahlhaus) sowie James Hepokoski und Warren Darcy (letztere bemühen sich bis zu einem gewissen Grad um eine historisch informierte Formbetrachtung, jedoch bilden sie dann eine eigene Systematik aus); 
3. theoriehistorische Primär- und weitere daran orientierte Sekundärliteratur. ${ }^{2}$

Aufbau und Inhalt des Buches orientieren sich an den verschiedenen Typen der Sonatentheorie von Hepokoski und Darcy 2006 (vgl. 18), erschöpfen sich jedoch nicht darin. An Heinrich Schenker ausgerichtete Strategien sind in dem Buch nicht tragend (unabhängig davon werden vereinzelt übergeordnete Gerüstsatzverläufe zur Veranschaulichung hinzugezogen). Zur wissenschaftlichen Untermauerung und für zusätzliche Informationen wird dem/der Leser*in weiterführende Literatur an die Hand gegeben.

Ein Glossar zu den verwendeten Formmodellen und Fachbegriffen am Ende des Buches bietet pädagogische Hilfestellung. Den jeweiligen Kapiteln sind außerdem zahlreiche Aufgaben zur weiteren Vertiefung beigegeben. Dies ist ein methodischer Vorteil gegenüber anderen Formenlehren, die solche Übungen normalerweise nicht anbieten. ${ }^{3}$ Die vielseitigen Aufgaben fordern dazu heraus, bestimmte Verallgemeinerungen nicht einfach starr und schablonenhaft anzuwenden, sondern mit dem neu Erlernten flexibel umzugehen, um die jeweiligen Werke an bestimmten Konventionen ihrer Zeit zu messen. Das Sujet fußt wie die amerikanischen Vorbilder auf dem ,klassischromantischen instrumentalen Repertoire und ist somit weitaus eingeschränkter als das deutschsprachiger Formenlehren. ${ }^{4}$ Dafür wird es jedoch viel differenzierter ausgeführt. Vokalformen, etwa des Liedes und der Oper, sind (bis auf den "Arientypus", siehe unten) weitgehend ausgeschlossen. Auch barocke Formen und Gestaltungsmittel werden nicht, wie es der

2 Etwa von Joseph Riepel, Heinrich Christoph Koch, Emanuel Aloys Förster, Georg Joseph Vogler, Anton Reicha, Carl Czerny, Robert Schumann sowie Budday 1983; Rosen 1988; Gjerdingen 2007 u. a.

3 Mir sind keine Lehrbücher mit hochschuldidaktischem Anspruch bekannt, die Aufgaben in dieser offenen Form anbieten. In wenigen werden zumindest weitere erklärte Beispiele angeboten, wie z. B. bei Kühn 1987 oder Dobretsberger 2016 und 2019.

4 Vgl. Dobretsberger 2016 und 2019; Altmann 2001; La Motte 1999; Kühn 1987 oder Leichtentritt 1927.
Untertitel nahelegt, eingehender thematisiert, sondern dienen eher zur schlaglichtartigen Beleuchtung bestimmter Traditionslinien der nachfolgenden Epochen. Die Ausführungen zur Formgestaltung romantischer Musik fungieren vornehmlich als Ausblick auf fortgeführte Gattungstraditionen der (Wiener) Klassik. So werden im Grunde die wichtigsten Instrumentalformen und Bauprinzipien zwischen etwa 1750 und 1850 in den Blick genommen, insbesondere mit einem Schwerpunkt auf der Sonate der Frühklassik bzw. des galanten und empfindsamen Stils (ca. 1750-70) und der Klassik ${ }^{5}$ (wie in den historischen Quellen werden hier Konvention und Innovation voneinander unterschieden). Gerade dieser Blick auf die frühen Gattungstraditionen erweist sich als besonders fruchtbar. Damit wird zweifellos ein tieferes Verständnis für Formstrategien späterer Epochen befördert und diese Perspektive ist gerade für deutschsprachige Formenlehren keineswegs selbstverständlich. Das im Buch erschlossene Korpus umfasst eine ausgewogene Mischung aus Klavier-, Kammer- und Orchestermusik mit den bekannten Standardwerken (meist durch erhellende Blickwinkel betrachtet) sowie eher unbekannteren Kompositionen. Vertreten ist ein großer Kreis an Komponist*innen, neben Mozart (Vater und Sohn), Haydn, Beethoven, Chopin, Mendelssohn u. a. auch Carl Philipp Emanuel und Johann Christian Bach, Muzio Clementi, Domenico Cimarosa, Leopold Koželuch, Clara Schumann u. a. ${ }^{6}$ - was durchaus zu einigen interessanten Neuentdeckungen führen kann.

Wie bereits erwähnt, werden ganz im Sinne der historischen Handwerkslehre ${ }^{7}$ (und in Anlehnung an gattungshistorische Hintergründe) die größeren Formen aus kleineren Basismodellen hergeleitet. Ausgangspunkt ist das Menuett bzw. die traditionelle zwei- und dreiteilige »binäre»

5 Die Epochengrenze liegt etwa um 1770, wobei sich bestimmte Phänomene natürlich zeitlich überschneiden können.

6 Einen guten Überblick gibt das Personenregister im Anhang des Buches. Auch Komponisten des späteren 19. Jahrhunderts wie Liszt, Mahler oder Bruckner sind gelegentlich einbezogen.

7 Vgl. etwa Riepel 1752/55 sowie Koch 2007. 
(Suitensatz-)Form. ${ }^{8}$ Ein weiteres Grundmodell, das erst im sechsten Kapitel zu den verschiedenen Rondoformen zum Tragen kommt, ist der an das barocke Rondeau angelehnte Reihungstyp. ${ }^{9}$ So dient das erste Kapitel als eine Art suniverse in a nutshell <, dem die größeren Formen als Sonaten- (bzw. Sinfonie-)sätze, Ouvertüren und Concerti durch bestimmte Erweiterungsstrategien entspringen. Diese induktive Methode ist klug, weil damit auch gewinnbringend auf den immer schon etwas verwirrenden (ursprünglich) Marx'schen Begriff der /Liedform verzichtet werden kann.

Während im ersten Kapitel (23-45) neben Menuett, Trio und Scherzo bereits der Bezug zu größeren zweigeteilt binären Formen veranschaulicht wird (namentlich »Scarlatti-Sonatenform « und "Ouvertürenform « bzw. »Arientyp «), kommt in den Folgekapiteln der dreigeteilt binäre Sonatensatz (Kammer- und Orchestermusik immer miteinbezogen) differenzierter zur Anschauung. Jedem einzelnen Formabschnitt des ,Sonaten(haupt)satzes، ist hier ein eigenes Kapitel zugeteilt. Wie in herkömmlichen Abhandlungen ${ }^{10}$ werden die einzelnen Formteile unter den traditionellen Überschriften »Exposition«, »Durchführung" und »Reprise" je für sich facettenreich erläutert. Im Kapitel zur Exposition (47-78) ist auch ein prägnanter Abschnitt zur »langsamen Einleitung" eingeschoben (73-77), welcher einen Formteil würdigt, der in der deutschsprachigen Formenlehre mitunter vernachlässigt wird.

Der Gedanke der kompositorischen Erweiterung und die Frage danach, was hinzukommen muss, damit aus der Miniatur größere Formkonzeptionen entstehen können, führt zunächst zum Koch'schen interpunktischen Prinzip formbildender Kadenzen in der Exposition. Hierzu werden Varianten vorgestellt und der Versuch, diese mit Entwicklungen nach 1770 und der Theorie der »medial caesura " von

8 Vgl. dazu die Ausführungen in Rosen 1988 und Hepokoski/Darcy 2006 oder auch SchmidtBeste 2006.

9 Siehe dazu auch die Ausführungen zum »Sonatentyp 4 « von Hepokoski/Darcy 2006, 388-396.

10 Vgl. etwa Rosen 1988, Caplin 1998, Hepokoski/Darcy 2006, Schmidt-Beste 2006, Amon 2011 u. a.
Hepokoski und Darcy zu vereinen. ${ }^{11}$ Dem folgen Erläuterungen und Diskussionen zu »Expositionen mit mehreren Zäsuren « (62 f.), "Monothematische[n] Expositionen« (63f.), "Strategien der Überleitung" zwischen Hauptund Seitensatz mit altem oder neuem thematischen Material (64-70), zur »DreitonartenExposition « (die sich historisch aus der Alternative zwischen den Nebentonarten III und $v$ in Moll entwickelt hat und später auch auf Durkontexte übertragen wurde, 70 f.) und zur $\triangleq E$ weiterung der formalen Dimensionen [der Exposition] im 19. Jahrhundert" (71-73). Nach dem erwähnten Exkurs über die langsame Einleitung folgt dann ein größeres Kapitel zur Themengestaltung, das sich vornehmlich an den altbekannten Formtypen "Satz « und »Periode" sowie an »Mischformen « orientiert und von dort aus zusätzliche weitere Themenformen ableitet. Gewinnbringend für den künstlerischen Satzlehreunterricht ist hier die Auflistung verschiedener typischer harmonischkontrapunktischer Gerüstsatzformeln zum Beginnen von `Sätzen .

Das Kapitel zur »Durchführung" (119-156) ist vornehmlich in zwei inhaltliche Kategorien aufgeteilt. Erstens wird die Durchführung als »)modifizierte Exposition «" mit deutlichem kadenziellen Absatz in einer Nebentonart als Konvention etwa zwischen 1750 und 1770 thematisiert (121 f.). Zweitens werden modernere Durchführungsweisen ab 1770 sehr genau hinsichtlich spezifischer Verlaufskurven mit motivisch-thematischer Arbeit, Sequenzen und weiteren kontrapunktischen Techniken in Augenschein genommen (125-138). Gebräuchliche Satzmodelle werden konkret aufgezeigt sowie typische harmonische Rückleitungsmöglichkeiten zur Reprise (147-154), was Leser*innen des Buches womöglich ebenfalls zum Selbstkomponieren und -improvisieren reizt.

Das Kapitel zur »Reprise« unter dem Motto "Neue Wendungen des Alten « (157-189) fokussiert spezifische Aspekte von Modifikation. Nach einem kurzen Blick auf bestimmte Einrichtungszwänge und -optionen in der "Transpositionsreprise« (160-163) werden verschiedene verallgemeinerbare Strategien der Rekomposition beleuchtet, die bei der Formgestaltung in ein sensibles Wechselspiel treten können: so die

11 Vgl. Hepokoski/Darcy 2006, 23-50. 
»Vermeidung kadenzieller und thematischer Redundanz« (163-170), die »Aufrechterhaltung des Bewegungsflusses" (171-174) und die "Aufrechterhaltung des proportionalen Gleichgewichts « der Gesamtform (174 f.). Schließlich werden auch »Reprisenanfänge abseits der Grundtonart" erläutert und mit schönen Beispielen ausgestattet (175-177). Es folgen Ausführungen zum "verschleierte[n] Reprisenbeginn « (177-181) und ein Ausblick auf das 19. Jahrhundert (181-184), mit besonderer Betonung auf dem »Verwischen formaler Grenzen zwischen Durchführung und Reprise«(181). Ein Kapitel zur "Coda" (184-188) rundet die gesamte Einheit zur 'Sonatenhauptsatzform ‘ ab.

Das anschließende Kapitel über das Rondo (191-210) behandelt, wie bereits erwähnt, vom barocken Rondeau-Prinzip ausgehend die bekannten Rondotypen, insbesondere Ketten- und Sonatenrondo. Dabei wird an Beispielen von Haydn und Mozart der Blick dafür geschärft, wann ein Rondo eher dem Reihungs- oder eher dem Sonatenprinzip verhaftet ist bzw. wie sich umgekehrt bestimmte Sonatenformen dem Rondoprinzip nähern (198-202, 204 f.). Interessant ist auch der Blick auf Haydns sinfonische Finali, die schwer einzuordnen sind und in denen fraglich wird, ob das Rondo überhaupt als feststehendes Formmodell oder nicht viel eher nur als Prinzip gelten kann (202204). ${ }^{12}$ Im Anschluss daran werden wie bei Hepokoski und Darcy die besonderen Rondi Carl Philipp Emanuel Bachs gewürdigt (205208). ${ }^{13}$ Ausblickend werfen die Autoren auch hier wieder ein Schlaglicht auf fortgeführte Gattungstraditionen im 19. Jahrhundert (208-210).

Wohl das lohnendste Kapitel für deutschsprachige Formenlehren mit didaktischem Anspruch stellt dasjenige zu Sonatenformen in Konzertsätzen dar (211-233). Anhand der rezeptartig verfassten Empfehlungen Georg Joseph Voglers und Heinrich Christoph Kochs wird die Genese von Konzertsatzformen verfolgt (211-213). Dabei bilden die Cembalokonzerte KV 107 W. A. Mozarts, in denen die Klaviersonaten op. 5 von Johann Christian Bach eingearbeitet sind, den Ausgangspunkt. Dies führt zu drei konventionellen Konzertsatztypen mit bestimmten Arten, Ritornelle an-

12 Vgl. dazu auch ebd., 413-415.

13 Vgl. ebd., 403. zuordnen (213-215). Anhand dieser Typologie wird dann ein Entwicklungsstrang mit anschaulichen Beispielen ausgerollt, der bis ins 19. Jahrhundert führt und spätere Werke auf diese Tradition hin beleuchtet (219 f.). Auch hier ergeben sich zahlreiche Schnittpunkte mit der historischen Kompositionspraxis, die sich für einen fortgeschrittenen Satzlehre- und Improvisationsunterricht sehr gut nutzen lassen. Mit diesem Kapitel, das der Vollständigkeit halber auch eine kurze Rückblende auf barocke Konzertsätze (217 f.) sowie weitere Ausführungen zur Reprisengestaltung im Verhältnis zur Soloexposition (219-225), zu Konzertsonatenformen in anderen Konzertsätzen (225 f., 227-229) sowie auf Grundlage anderer Sonatentypen (226 f.) enthält, erfüllen Diergarten und Neuwirth ein dringliches Desiderat. Gerade Musiklehrer*innen dürfte diese prägnante deutsche Zusammenfassung und Erweiterung von Hepokoskis und Darcys Darstellung der Konzertsatzform für ihren Schulunterricht sehr entgegenkommen.

In einem Ausblick (235-252) bekennen die Autoren nochmals ihre einführend erklärte Haltung, die vorgestellten Formtypen eher als Bündel charakteristischer Module und übergreifender Prinzipien und nicht als rigide Schemata zu begreifen. So wird abschließend mit dem durch das Buch hinzugewonnenen Wissen aufgezeigt, wie ein solch flexibler »modularer « Analyseansatz auf Fallbeispiele angewendet werden kann, die sich zunächst einer eindeutigen Zuordnung entziehen (etwa der Schlusssatz aus W. A. Mozarts populärer Serenade KV 525, Klaviersonaten Muzio Clementis und frühe Streichquartette Joseph Haydns, 237-245). Durch die Diskussion von unterschiedlich kombinierbaren Merkmalen und Strategien der verschiedenen historischen Sonatensatztypen (die als Stereotype immer nur als Annäherung zu verstehen seien) könne man zu plausiblen Deutungen finden (235-237). Zum Schluss erinnern die Autoren an die bekannten allgemeinen Grundkriterien der Erfassung klassischer Formen, wie etwa "Thematik", "Tonalität«, »Interpunktion«, »Wiederholung", "Wiederkehr", "Transposition «, "Variation «, "Kontrast" usw. (251), um dem Spannungsverhältnis von Modell und individueller Werkgestalt in letzter Konsequenz gerecht zu werden. 
So hat man also am Ende des Buches eine weitere, längst fällige Runde im hermeneutischen Zirkel der deutschsprachigen Formenlehre zurückgelegt, hat neuerlich wichtige Aspekte der Gattungshistorie um 1750 und eine bekömmliche Portion aktueller angloamerikanischer Musiktheorie mit im Gepäck - und doch ist man wieder in der schützenden Nähe des sväterlichen` Dahlhaus'schen Denkens mit der dort gebotenen Vorsicht vor Verallgemeinerungen ${ }^{14}$ angelangt. Dennoch oder vielleicht gerade dafür hat sich diese Runde zweifelsfrei gelohnt.

Im Hinblick auf mögliche Folgepublikationen seien nachstehend noch einige Punkte diskutiert. So sehr sich die neue Formenlehre durch ihren integrativen, wissenschaftlich reflektierten Ansatz und auch durch ihr zusammenfassendes und geordnetes Vorgehen auszeichnet, finden sich darin auch einige methodische und pädagogische Inkonsequenzen. Dies dürfte gerade für Anfänger*innen und für ein eigenständiges Lernen snach Buchı, jedoch auch für das Fachpublikum noch einige Fragen offenlassen.

So fällt das Kapitel über die "Strategien der Themengestaltung" (79-117) gegenüber den anderen Darstellungen insofern deutlich schwächer aus, als hier plötzlich nicht wie sonst von der Theorie und Praxis frühklassischer bzw. galanter Sonatentradition ausgegangen wird, sondern von den althergebrachten Formenlehrebegriffen des 20. Jahrhunderts, nämlich ausschließlich von "Satz " und "Periode" (welche sich bekanntlich an Musik ab etwa 1770 ausrichten). So vermittelt das Kapitel leicht den anachronistischen Eindruck, dass alle anderen Formen und thematischen Gestalten daran per se zu messen und davon abzuleiten seien. Gerade für die innovative Haltung des Buches wäre es jedoch konsequenter gewesen, die Entwicklung des Themenbegriffs ebenfalls historisch nachzuverfolgen. Was konnte in der Zeit des frühen Haydn oder Mozart überhaupt ein 'Themar sein, wie zeigt sich das in der Musik und wie ändert sich das im Laufe der Zeit bis ins 19. Jahrhundert? Falls sich hierfür nicht genügend Anhaltspunkte in der Historie böten, wäre es auch interessant, die Diskussion auf Grundlage alternativer Konzepte etwas offener zu führen. Auch instrumentatorische Aspekte, wie sie etwa Michael Polth für Sinfonieexpositionen des 18. Jahrhunderts herausgearbeitet hat, ${ }^{15}$ wären für eine solche Themendiskussion durchaus von Interesse. Folglich wäre dann auch die Frage nach thematischem Komponieren überhaupt (je nach IThema Definition) für bestimmte Kompositionen zu stellen und mit der Frage nach Reprisenerlebnissen zu verknüpfen.

Ein solcher Blick wäre nicht nur für die aktuelle Forschung, sondern auch für die Lehre insofern lohnend, als er weitere Mittel an die Hand gäbe, Themen flexibler definieren und erkennen zu können. Mit einer unabhängigeren Sichtweise könnten dann auch endlich solch unglückliche Bezeichnungen wie die »Mischform [aus Satz und Periode] ", welche als Thementyp mindestens ebenso häufig auftaucht wie Satz und Periode selbst, hinterfragt und eventuell sogar gänzlich aufgegeben werden, ähnlich, wie es ja auch mit der Liedform und anderen Begriffen oder Perspektiven im vorliegenden Buch immer wieder geschieht. ${ }^{16}$ Die anderen, teils im Buch genannten Thementypen $(104,110)$ wären folglich gleichberechtigter zu behandeln. Mit solch neuen Perspektiven könnte die Themenanalyse wieder mehr zu einer spannenden musikanalytischen Frage werden.

Zur Darstellung im Einzelnen sei außerdem angemerkt, dass für das Erkennen von Themenformen nach Caplin ebenfalls nicht klar genug Hilfestellung gegeben wird. Es wird lakonisch darauf verwiesen, dass eine »Kadenz « ein solches (Caplin'sches) Thema beschließt. Welche Art von Kadenz das im jeweiligen Kontext sein kann, müsste hier genauer erläutert werden. Ein Verweis auf dafür ebenfalls relevante Ausführungen

15 Vgl. Polth 2000.

16 Hierbei könnte man allerdings auch noch entschiedener vorgehen - wie z. B. bei der Auseinandersetzung mit dem Konzept »Monothematik « $(63 \mathrm{f}$.). Dass in diesem Zusammenhang zwar der Begriff kritisiert, aber dann doch wieder selbstverständlich aufgegriffen wird, leuchtet nicht ganz ein, ebenso wenig wie die Tatsache, dass in diesem Zusammenhang nicht auch auf das Gebot der > Einheit in der Mannigfaltigkeit` bzw. sMannigfaltigkeit in der Einheit` verwiesen wird (vgl. Koch 2007, 263; Gerhard 2002, 179 f.). 
an anderer Stelle (56) und entsprechende Erläuterungen im Glossar wären hier zudem nützlich.

Einige terminologische Unschärfen könnten dazu führen, dass Anfänger*innen nicht klar wird, wann von den Themen im Besonderen und wann von entsprechend geformten musikalischen Verläufen im Allgemeinen die Rede ist, insbesondere im Kapitel zum "Satz« (vgl. dazu 84-88). Es wäre hier von Nutzen, nichtthematische Formfunktionen (welche eigentlich auch nicht zur Kapitelüberschrift passen) nicht mit »Satz « zu benennen, sondern als ssatzartig ‘ oder sdem Satzprinzip entsprechend zu bezeichnen. Umgekehrt wäre, gerade im Hinblick auf die Themen-Definition nach Caplin, im ersten Kapitel der Begriff »thematisch« (25 f.) durch >motivisch (o. ä.) zu ersetzen. Auch der etwas lapidare Verweis auf das "Ratz'sche" dreiteilige Lied (83) und der trockene Hinweis auf das Kapitel zur zwei- und dreiteiligen binären Form (109) ist insofern nicht befriedigend, als dieser Gedanke dort nicht in Bezug auf Themengestalten erläutert wird. Dass bzw. inwiefern ein Thema einer solchen Kleinform entsprechen kann, ist für Anfänger*innen ohne konkrete Illustration schwer nachvollziehbar. Möchte man dem gerecht werden, müssten im ersten Kapitel die binären Grundformen auch in Bezug auf die Themengestaltung konsequent weitergedacht und veranschaulicht werden.

Im Gegensatz zum klaren und übersichtlichen Gesamteindruck des Buches wirken die Ausführungen zur musikalischen Interpunktion der Exposition nach Koch in Verbindung mit Anton Reichas Themenbegriff und der Diskussion um die Mittelzäsur etwas verschwommen (47-60). Einige der gegebenen Beispiele und Aufgaben, wie z. B. jene zu KV 279 und 280 (53), passen nicht recht zu den vorangegangenen Erklärungen. ${ }^{17}$ Zudem ist die integrative Darstellung der Ansätze etwas verwirrend. Die hervorgehobene Nummerierung in Reichas Zitat (55) passt inhaltlich nicht, wie zunächst sugge-

17 Die zuvor erläuterten Abweichungen zu Kochs »Hauptform« (Aussparen des Ganz- oder Halbschlusses in der Grundtonart, Vervielfältigen von Absatzformeln in der Nebentonart) entsprechen nicht jenen, die offenbar in den Aufgaben vermittelt werden sollen (Aussparen/Überspielen eines prägnanten Halbschlusses in der Nebentonart). riert wird (55-57), zu Kochs vierteiliger »Hauptform « in der Tabelle, welche die Mittelzäsur in Beziehung zu Kochs Kadenzen zeigt (59).

In Reichas Aufzählung (55) ${ }^{18}$ kommt das »zweite Thema « an dritter Stelle, an zweiter Stelle steht eine modulierende »Brücke» (entsprechend wäre hier der Ort für einen deutlichen Halbschluss in der neuen Tonart). Die vorausgegangenen Erklärungen zu Kochs Grundmodell und ihren ersten beiden Teilen umfassen jedoch nur Ganzschluss und Halbschluss in der Grundtonart (Letzterer im Sinne des >bifocal close «). Die weiteren Ausführungen (56f.) stellen dennoch zunächst unvermittelt eine Analogie zwischen Koch und Reicha aufgrund der ersten beiden Teile her, was immerhin plausibel erscheint. Jedoch entspricht dies wiederum nicht der Tabelle (59). Hier wird Kochs Form auf andere Weise integriert. Der Ort der Mittelzäsur, der in Bezug auf die Gegenüberstellung von Koch und Reicha eigentlich am Ende des zweiten Teils markiert sein müsste, ist plötzlich an dritter Stelle der Koch'schen Hauptform vermerkt (konsequenterweise beim Halbschluss in der Tonart der V. Stufe). Dieses Vorgehen ist verwirrend und insofern auch schwer nachvollziehbar, weil eine modulierende Passage inhaltlich nicht einem dritten Teil der Koch'schen Grundform entsprechen kann. Dieser ist aus der kompositorischen Tradition heraus (im Gegensatz zur Meinung der Autoren) durchaus definiert (wenn auch nicht sthematisch ‘). Bei Koch ist ja die neue Tonart schon vorher quasi unvermittelt durch den sbifocal closes herbeigeführt worden, d. h. der dritte Teil steht bereits in der neuen Tonart, hat also weitaus mehr Stabilität als eine modulierende Passage. Außerdem würde eine solche Form, so, wie sie die erste Tabellenspalte suggeriert, in der Regel nicht nur die Schlusskadenz, sondern noch weitere Kadenzen nach sich ziehen und damit Proportionen ausbilden, die ebenfalls nicht einfach zur Koch'schen Grundform, sondern mehr zu Reichas Modell (oder der oben genannten Analogie) passen. Hier müsste man dann von Varianten oder Erweiterungen des Koch'schen Modells ausgehen, was schnell unübersichtlich wird und in dieser Perspektive grundsätzlich auch fragwürdig ist. Vielleicht wäre es hier die Überlegung wert, ob nicht eine offenere, weniger auf eine Synthese von Koch, Reicha und Hepokoski und Darcy abzielende Darstellung dem »modularen« Ansatz der Formenlehre mehr Rechnung tragen könnte.

Die »modifizierte Exposition«(121-123) wird ausschließlich in Bezug auf Dursätze beschrieben. Die damit automatisch aufkommende

18 Vgl. auch Reicha 1832, 1159-1161. 
Frage nach entsprechenden Mollsätzen bleibt unbeantwortet.

Für Anfänger*innen sind zudem die Abschnitte zur Ouvertüren- und Arienform ein wenig irreführend (38-41). Geht man davon aus, dass das Buch auch als Nachschlagewerk für einzelne Themenbereiche dienen soll, könnte leicht der Eindruck entstehen, dass Ouvertüren ausschließlich aus der hier beschriebenen »Ouvertürenform« bestehen, was allein schon für die Wiener Klassik nicht zutrifft. Hier wären entsprechende Verweise auf das letzte Kapitel (245-248), weitere Ausführungen und ein Ausblick auf das 19. Jahrhundert empfehlenswert. Auch bei der Beschreibung der "Arienform« sollte zumindest ein Hinweis auf andere Formmodelle der Arie gegeben werden. Außerdem sollten dort auch einige Arien als Beispiele dienen und nicht nur ein Verweis auf das entsprechende Kapitel in Charles Rosens Sonata Forms gesetzt werden (39, 44, Anm. 20), der für ein erweitertes Lesepublikum eher unbrauchbar ist. Zur Illustration der sogenannten "Scarlatti-Sonatenform《 (35-38) wäre es ebenfalls ratsam, zumindest ein Beispiel von Domenico Scarlatti auch anzuführen.

In Bezug auf Rezeption und Würdigung fällt auf, dass zwar prägenden amerikanischen $\mathrm{Mu}$ siktheoretikern wie Rosen, Caplin oder Robert Gjerdingen sowie salten Größen ‘ wie Ratz oder Dahlhaus ein Platz im Autorenverzeichnis zugestanden wird, nicht jedoch weiteren, für das fachlich-pädagogische Anliegen einflussreichen Theoretikern und Musikforschern deutschsprachiger Provenienz (etwa Kühn oder Wolfgang Budday).

Ob man das Fehlen übergeordneter Schenker'scher Ansätze als Vor- oder Nachteil betrachten will, soll an dieser Stelle nicht weiter vertieft werden. Zumindest würde dies zusätzliche Ausführungen, gerade auch in Bezug auf die im Buch besprochenen Satzmodelle verlangen. ${ }^{19}$ Dass die Autoren darauf verzichten, ist also durchaus nachvollziehbar, die Gründe hierfür sollten jedoch auch genannt werden.

Wie sich bereits zeigte, bietet die produktionsorientierte Perspektive des Buches nicht nur für einen analytischen, sondern auch für einen historisch orientierten künstlerischen Tonsatzunterricht Impulse, da formale Strategien vorgestellt werden, die zur kompositorischen Umsetzung ,förmlich einladen`. Im Sinne einer Durchdringung der einzelnen Fachbereiche und einer konsequent verfolgten historischen Satzlehre wäre es dann jedoch auch wünschenswert, noch mehr praktische Aufgaben und Hinweise für Stilkopien an die Hand zu bekommen und nicht fast ausschließlich aus betrachtender Distanz an das Gebiet 'Formenlehre heranzugehen. Damit erschlössen sich weitere Kategorien, die sich mit dem Bereich künstlerischer Forschung überschneiden, andere hingegen träten vielleicht mehr in den Hintergrund. Dass dies jedoch nicht die Aufgabe eines einzigen Buches sein kann und dass das vorliegende hierfür bereits wichtige Anreize gibt, daran besteht kein Zweifel. So kann man nur auf eine stete Erweiterung der Laaber-Reihe hoffen - auf Darstellungen, die nicht ausschließlich dem althergebrachten und in sich separierten Kanon frönen, sondern die auch, ganz im Sinne des besagten hermeneutischen Zirkels und in Memoriam unseres vor zehn Jahren verstorbenen Kollegen Diether de la Motte, weitere Epochen, Formen und Techniken für eine in sich konsistente Satzlehre bis hin ins 20. und 21. Jahrhundert erschließen.

Die oben genannten Kritikpunkte sind insgesamt nicht sehr gravierend. Fraglos eröffnet das Buch lohnende Perspektiven, womit es hoffentlich gelingen wird, die internationale Formenlehrediskussion der vergangenen drei Jahrzehnte mehr in eine breitere deutschsprachige Öffentlichkeit hineinzutragen. Dies würde zu einer aufgeklärteren, erweiterten Fachdiskussion beitragen, von der deutschsprachige Hochschulen und Universitäten und so auch die Lehrerbildung profitieren könnten. All das wäre also die größte Errungenschaft des Buches, was sich aber hoffentlich schon früher als erst nach 30 Jahren zeigen wird.

Juliane Brandes 


\section{Literatur}

Altmann, Günter (2001), Musikalische Formenlehre. Ein Handbuch mit Beispielen und Analysen. Für Musiklehrer, Musikstudierende und interessierte Laien [1970], 8. Auflage (überarbeitete Neuauflage), Mainz: Schott.

Amon, Reinhard (2011), Lexikon der musikalischen Form. Nachschlagewerk und Fachbuch über Form und Formung der Musik vom Mittelalter bis zur Gegenwart, in Zusammenarbeit mit Gerold Gruber, Wien: Doblinger.

Budday, Wolfgang (1983), Grundlagen musikalischer Formen der Wiener Klassik. An Hand der zeitgenössischen Theorie von Joseph Riepel und Heinrich Christoph Koch dargestellt an Menuetten und Sonatensätzen (1750-1790), Kassel: Bärenreiter.

Caplin, William E. (1998), Classical Form. A Theory of Formal Functions for the Instrumental Music of Haydn, Mozart, and Beethoven, New York: Oxford University Press.

Dahlhaus, Carl (2001), "Zur Theorie der musikalischen Form « [1977], in: Allgemeine Theorie der Musik II (= Gesammelte Schriften in zehn Bänden, Bd. 2), hg. von Hermann Danuser in Verbindung mit HansJoachim Hinrichsen und Tobias Plebuch, Laaber: Laaber, 284-300.

Dobretsberger, Barbara (2016), Formenlehre: Formen der Instrumentalmusik. Ein Handbuch für Studierende und andere Neugierige, 2. Auflage, Wien: Doblinger.

Dobretsberger, Barbara (2019), Formenlehre: Formen der Vokalmusik. Ein Handbuch für Studierende und andere Neugierige, Wien: Doblinger.

Froebe, Folker (2015), „On Synergies of Schema Theory and Theory of Levels. A Perspective from Riepel's Fonte and Monte«, Zeitschrift der Gesellschaft für Musiktheorie 12/1, 9-25. https://doi.org/10.31751/802 (24.4.2020)

Gerhard, Anselm (2002), London und der Klassizismus in der Musik. Die Idee der "absoluten Musik" und Muzio Clementis Klavierwerk, Stuttgart: Metzler.
Gjerdingen, Robert (2007), Music in the Galant Style, New York: Oxford University Press.

Hepokoski, James / Warren Darcy (2006), Elements of Sonata Theory. Norms, Types, and Deformations in the Late-Eighteenth-Century Sonata, New York: Oxford University Press.

Koch, Heinrich Christoph (2007), Versuch einer Anleitung zur Composition [1782; 1787; 1793], Studienausgabe, hg. von Jo Wilhelm Siebert, Hannover: Siebert.

Kühn, Clemens (1987), Formenlehre der Musik, Kassel: Bärenreiter.

La Motte, Diether de (1999), Musik Formen. Phantasie, Einfall, Originalität. Ins Ohr springend, für Aufmerksame hineinversteckt, Augsburg: Wißner.

Leichtentritt, Hugo (1927), Musikalische Formenlehre [1911], 3., beträchtlich erweiterte Auflage, Leipzig: Breitkopf \& Härtel.

Marx, Adolph Bernhard (1838), Die Lehre von der musikalischen Komposition praktisch-theoretisch, Bd. 2, Leipzig: Breitkopf \& Härtel.

Polth, Michael (2000), Sinfonieexpositionen des 18. Jahrhunderts. Formbildung und Ästhetik, Kassel: Bärenreiter.

Ratz, Erwin (1973), Einführung in die musikalische Formenlehre. Über Formprinzipien in den Inventionen und Fugen Johann Sebastian Bachs und ihre Bedeutung für die Kompositionstechnik Beethovens, 3. Auflage, Wien: Universal Edition.

Reicha, Anton (1832), Vollständiges Lehrbuch der musikalischen Komposition, hg. und übers. von Carl Czerny, Bd. 4, Tle. 8-10, Wien: Diabelli.

Riepel, Joseph (1752/55), Anfangsgründe zur musicalischen Setzkunst. Nicht zwar nach alt-mathematischer Einbildungs-Art der Zirkel-Harmonisten/ Sondern durchgehends mit sichtbaren Exempeln abgefasset, Tle. 1 und 2, Regensburg: Bader / Frankfurt a. M.: ohne Verlagsangabe.

Rohringer, Stefan (2015), »Schemata und Systemcharakter", Zeitschrift der Gesellschaft für Musiktheorie 12/1, 27-68. https://doi.org/ 10.31751/841 (24.4.2020) 
Rosen, Charles (1988), Sonata Forms, 2. Auf- Schoenberg, Arnold (1967), Fundamentals of lage, New York: Norton.

Schmidt-Beste, Thomas (2006), Die Sonate. Geschichte - Formen - Ästhetik, Kassel: BärenMusical Composition, hg. von Gerald Strang und Leonard Stein, London: Faber and Fareiter. ber.

Brandes, Juliane (2020): Felix Diergarten / Markus Neuwirth, Formenlehre. Ein Lese- und Arbeitsbuch zur Instrumentalmusik des 18. und 19. Jahrhunderts, Laaber: Laaber 2019. ZGMTH 17/1, 167-175.

https://doi.org/10.31751/1034

(C) 2020 Juliane Brandes (juliane.brandes@moz.ac.at)

Universität Mozarteum Salzburg [University Mozarteum Salzburg]

Dieser Text erscheint im Open Access und ist lizenziert unter einer Creative Commons Namensnennung 4.0 International Lizenz.

This is an open access article licensed under a

Creative Commons Attribution 4.0 International License.

eingereicht / submitted: 10/04/2020

angenommen / accepted: 10/04/2020

veröffentlicht / first published: 15/06/2020

zuletzt geändert / last updated: 15/06/2020 